\title{
PLASMONICS IN SEMICONDUCTORS AND IN Ag SUBMICRON WIRES AND GRAPHENE
}

\author{
H. Hartnagel, S. Al-Daffaie, and O. Yilmazoglu \\ Technical University Darmstadt, Merckstr. 25, D 64283 Darmstadt, Germany \\ E-mail: hartnagel@mwe.tu-darmstadt.de
}

Received 31 July 2015; revised 5 November 2015; accepted 15 December 2015

\begin{abstract}
Some 50 years ago discussions of plasmonics in semiconductors lead to many new concepts such as travelling domain structures with applications leading even to logic systems. Now plasmonics of submicron wires of Ag and graphene bring new device concepts for the fabrication of compact THz sources and optical focusing of the beat signal into the active area. Here as an experimental example such new opportunities are involved with compact THz sources based on optical laser mixing. They include resonant plasmonic structures at $\mathrm{THz}$ and optical frequencies to locally enhance the electromagnetic fields at $\mathrm{THz}$ as well as optical frequencies by the combination of semiconductor-graphene plasmons respectively by the semiconductor-metal-nanostructure plasmons.

Of particular interest is the usage of graphene, which is optically transmitting and which is either a semimetal or can be transformed into a semiconductor by reducing the width of its strips to about $30 \mathrm{~nm}$, opening a band gap in the meV to tens of the meV range.

A successful experimental structure for continuous-wave THz photomixing is fabricated using 1D and 2D nanocontacts either on low-temperature-grown (LTG) GaAs or on nitrogen ion-implanted $\left(\mathrm{N}^{+} \mathrm{i}\right) \mathrm{GaAs}$ and graphene sheets. The overlaying 1D and 2D nanocontacts were formed by silver nanowires with a diameter of 60 or $120 \mathrm{~nm}$. They can handle currents of $>10$ and $>30 \mathrm{~mA}$, respectively, without electromigration enabling reliably high photocurrents and field enhancement at $\mathrm{THz}$ frequencies by plasmonic effects.

The nanomaterial structurization in connection with present-day plasmonic applications is now to be discussed in a similar manner as past opportunities with semiconductor plasmonics.
\end{abstract}

Keywords: collective excitations, radiowave and microwave technology, nanowires, electronic transport in graphene PACS: 73.20.Mf, 84.40.-x, 62.23.Hj, 72.80.Vp

\section{Introduction}

New device concepts and materials for the fabrication of compact $\mathrm{THz}$ sources are of particular interest. Many applications ranging from imaging, space communications, quality control, and homeland security require $\mathrm{THz}$ waves at sufficient power from reliable sources. Such compact $\mathrm{THz}$ sources usually include optical laser mixing, Schottky diodes, varactor diodes for frequency multiplication, tunnelling diodes, unitravelling-carrier photodiodes or quantum cascade lasers [1].

The frequency ranges of $\mathrm{THz}$ have numerous interactive involvements such as $\mathrm{THz}$ signal generation by optical mixing of two light frequencies. The opportunities of both $\mathrm{THz}$ and optical waves to be enhanced by interaction with plasmonics are well known. Both resonant and overdamped plasma waves enable new $\mathrm{THz}$ devices such as sources, detectors and mixers.

Then an experimental effort is undertaken correspondingly. To enhance optical penetration into the nonlinear semiconductor such as GaAs, Ag nanometric-sized wires are employed [2-4]. The use of graphene as an optically transparent and electrically conducting layer is additionally of interest due to the existence of relevant $\mathrm{THz}$ plasmons [5, 6.

This work reminds one of a considerable effort of semiconductor plasma instabilities [ [ ] in the past. Originally, plasma effects occurred in gaseous plasmas. Then one considered the instability concept in semiconductors with electron and hole plasmas, 
where additional features occurred. They depend largely on an interaction with the crystal. This can, for example, result in a change of the effective mass of the charge carriers involved. Often, a negative slope of relevant parameters causes instabilities. An example is the transferred-electron effect, in its simple form called the Gunn effect.

The relevant book of one of the present authors (Hartnagel) [⿰] was largely discussed and Prof. Juras Požela considered even its translation into the Russian language at that time. Therefore this author and his colleagues are very happy to dedicate this article to the deceased Prof. Požela on the occasion of his 90th birthday.

Using bulk semiconductors, a number of plasmonic studies were reported recently such as the cases [8-10]. In the past [ [ of material structurizations and their resulting processes, such as the transferred-electron effect, avalanching or various types of heterojunctions giving plasma resonances or even plasma domain formations. Such domain dynamics lead previously even to various types of logic-system capabilities or modulation techniques for communication applications. It is suggested here that such possibilities should be considered along nanometric dimensions on $\mathrm{Ag}$ wires or along the two-dimensional sheets of graphene in order to obtain specialist capabilities such as the sharpening of the narrow-band.

\section{The experimental structure of the realized plasmonic system}

The plasma behaviour is based on its resonance frequency. There are a number of features of the solid materials, which affect this resonance. In the experimental system, where plasmonics can be particularly effective, one uses sub-micron Au or Ag wires to enhance the focussing of optical signals into underlying semiconducting materials, as has been used successfully with solar cells. Similarly, graphene can be employed to enhance the emission of $\mathrm{THz}$ waves out of the semiconductor. Here the semiconducting material is low-temperature-grown (LTG) GaAs or nitrogen-implanted $\left(\mathrm{N}^{+} \mathrm{i}\right) \mathrm{GaAs}$. This is covered on one of the open wafer surfaces by a graphene sheet. In particular, this allows electrical conduction and optical transmission. Metal electrodes of very narrow conducting lines allow the use of optical-frequency light for $\mathrm{THz}$ generation in the LTG or $\mathrm{N}^{+} \mathrm{i}$ $\mathrm{GaAs}$ for optical illumination. It is necessary not to have only a few of such wires spread over the semiconductor structure but to space them in an appropriate regularity. This requires the separation of the wires from each other by careful consideration of the plasmonic distances outside the wire, particularly into the semiconductor. The existence of an intermediate thin graphene layer of one or even several atomic thicknesses does not influence these
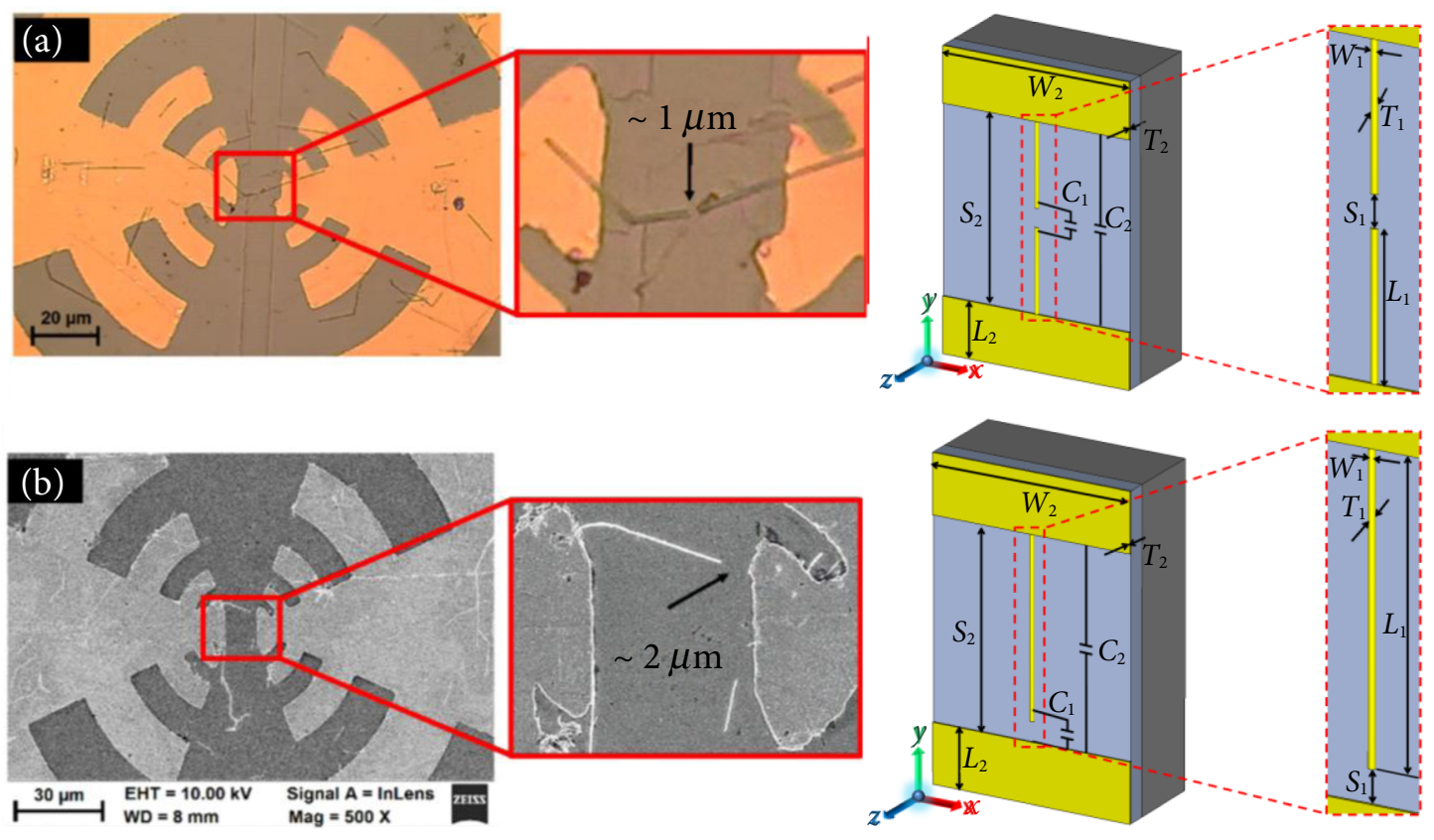

Fig. 1. Ag-NW photomixer, (a) optical microscope picture of a tip-tip Ag-NW photomixer and an equivalent model for capacitance calculation, (b) SEM picture of a tip-contact Ag-NW photomixer and an equivalent model for capacitance calculation. 
effects particularly. These permit the creation of $\mathrm{THz}$ antennas with enhanced signal emission [11]. On top of this, Ag wires of 60 or $120 \mathrm{~nm}$ diameter are deposited in order to enhance the two optical laser signals penetrating into the semiconductor for enhanced optical mixing to obtain $\mathrm{THz}$ signals due to the difference of the optical frequencies as shown in Fig. 1] [1].

A broadband log-periodic-antenna was fabricated with a standard optical lithography process on low-temperature-grown (LTG) GaAs or on nitrogen ion-implanted $\left(\mathrm{N}^{+} \mathrm{i}\right) \mathrm{GaAs}$. Afterwards, a simple spin coating method was used to distribute a commercially available Ag-NW over the wafer. The diameter varied only slightly for the commercial nanowires. The nanowires were randomly distributed and have different positions. The investigated photomixer in Fig. 1 (a) had a nanowire diameter of $120 \mathrm{~nm}$ and a tip-tip gap of $1 \mu \mathrm{m}$. An alternative and simpler configuration was realised using a single $\mathrm{Ag}-\mathrm{NW}$ with a diameter of $120 \mathrm{~nm}$ connected to one of the DC contacts. The gap to the second DC contact (tip-contact) was $2 \mu \mathrm{m}$ (Fig. 1(b)) [11]. Noble nanomaterial plasmonics such as Ag-NW and graphene can enhance the $\mathrm{THz}$ field and focus the optical beat signal into the active area.

\section{The plasmonic effect of graphene}

The generation of plasmonic resonances in graphene is possible at $\mathrm{THz}$ frequencies. This is due to the semi-metal properties of graphene. It is, of course, also possible to have semiconducting efforts by creating graphene strips. In such structures a number of electronic concepts were proposed, as shown in Fig. 2 [12], which also can be combined with plasmonic concepts as indicated in this manuscript.

There, such ideas as quantum cascading were considered for the generation of $\mathrm{THz}$ signals. Such concepts could then be employed to have special resonating concepts for the generation of particular $\mathrm{THz}$ properties such as very narrow-band spectra or signals of reduced noise, in particular if ballistic effects are to be exploited as shown in Fig. 3 [13.

\section{The experimental behaviour}

The generation of $\mathrm{THz}$ signals from such new plasmonic devices is highly successful and exceeds the results with similar structures without graphene and nanometric-sized wires [11]. The new concepts are highly stable, as shown in Figs. 4 and 5 [11]. Here, to verify the potential of 1-D and 2-D nano-

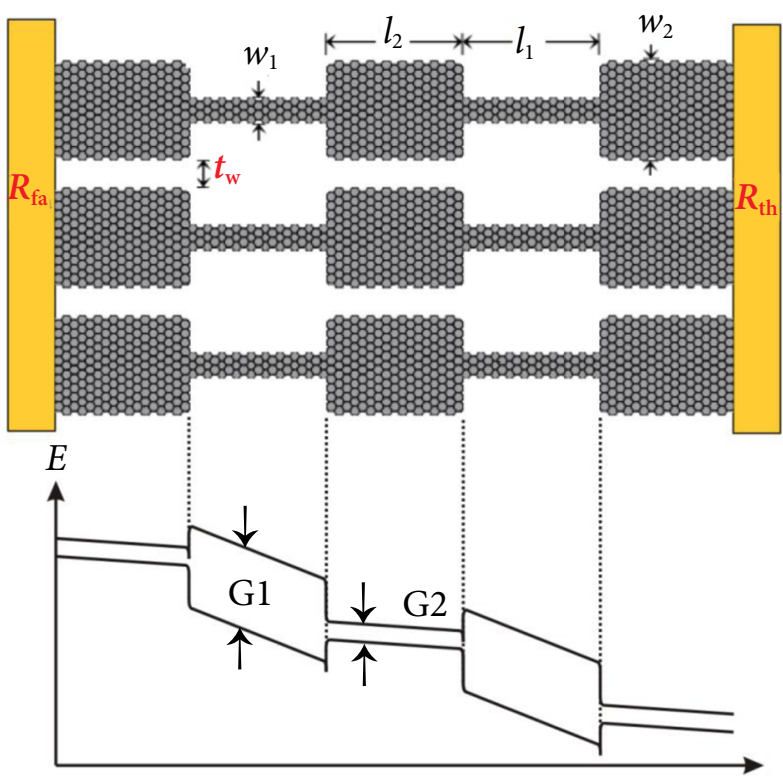

Fig. 2. The real space (top) and band diagram (bottom) under bias for a ballistic graphene resonator structure. The design data: $\mathrm{G} 1=200 \mathrm{meV}$ and $\mathrm{G} 2=40 \mathrm{meV}$, $w_{1}=17 \mathrm{~nm}$ and $w_{2}=21 \mathrm{~nm}, l_{2}=v_{0} / f, v_{0} \approx v_{\mathrm{F}} \approx 10^{6} \mathrm{~m} / \mathrm{s}$; $v_{0}$ is the electron velocity, $v_{\mathrm{F}}$ is the Fermi velocity in graphene, $f$ is terahertz frequency; for $f=1 \mathrm{THz}: l_{2}=1 \mu \mathrm{m}$, $l_{1}=t_{\mathrm{w}} \approx l_{2} / 3$.
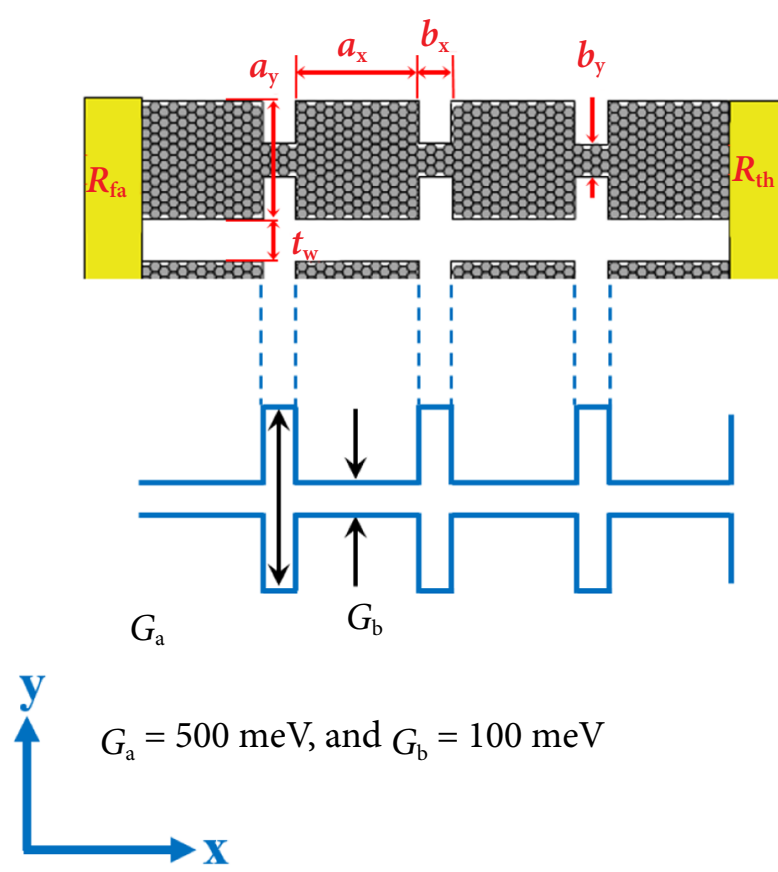

Fig. 3. The design of the structure for $\mathrm{THz}$ emission. The design data: $R_{\mathrm{fa}}$ and $R_{\mathrm{fb}}$ are the lower frequency biases, $a_{y}=200 \mathrm{~nm}, b_{y}=40 \mathrm{~nm}, a_{x}=V_{0} / f$, and $V_{0} \approx V_{\mathrm{F}} \approx 10^{6} \mathrm{~m} / \mathrm{s}$. $V_{0}$ is the electron velocity, $V_{\mathrm{F}}$ is the Fermi velocity in graphene, and $f$ is the terahertz frequency; for $f=1 \mathrm{THz}$ : $a_{x}=1 \mu \mathrm{m}$, and $b_{x}=t_{\mathrm{w}} \approx a_{x} / 3$. 
contacts as photomixer electrodes, the $\mathrm{THz}$ signal versus frequency was measured using the Golay cell bolometer and compared with the conventional photomixer with 6-finger electrodes under the same measurement conditions. The $\mathrm{THz}$ signal was focused onto the Golay cell window using a Si lens and two parabolic mirrors. The output of the Golay cell fed the lock-in-amplifier to measure the relative $\mathrm{THz}$ signal. A free space mechanical chopper was used to modulate one of the optical lasers with $20 \mathrm{~Hz}$ chopping frequency. Thus, the nanowire and the interdigital photomixers were on the same $\mathrm{N}^{+} \mathrm{i}$ GaAs wafer, had the same antenna structure, and measurement environment. This method was also applied to the graphene photomixer where the same LTG GaAs wafer was used for the multi-layer graphene (MLG) and the interdigital photomixers. A very clear improvement is shown by measurement results due to the use of Ag-NW electrodes instead of the interdigital electrodes, where the relative power and frequency increased in the upper part of the $\mathrm{THz}$ measurement range up to $1.9 \mathrm{THz}$; see Fig. 4 .

The MLG photomixer measurement results showed a clear improvement in the $\mathrm{THz}$ power but a slight im- provement in the maximum frequency, as shown in Fig. 5 [11]. The MLG photomixer has an increased photocurrent and a reliable operation, whereas there is no significant improvement in the frequency due to the comparable capacitance in both photomixers.

\section{Conclusions}

Ag-NW and graphene represent a promising nanomaterial for new plasmonic devices. Plasmonic contact nanoelectrodes can enhance quantum efficiency and ultrafast operation compared to conventional photomixers. An example of this field is presented here in connection with THz-generation by optical mixing. The Ag-NW electrode with a physical diameter of $120 \mathrm{~nm}$ provides a device capacitance of $0.1 \mathrm{fF}$, which is more than ten times lower compared with interdigital six-finger electrodes. The combination of a very low capacitance and a high photocurrent allowed the use of the upper part of the $\mathrm{THz}$ range, $2 \mathrm{THz}$ with a sufficient signal-to-noise ratio for a broad spectral range. The available $\mathrm{THz}$ measurement setup showed already $1.9 \mathrm{THz}$, which was only limited by the laser tunability and antenna
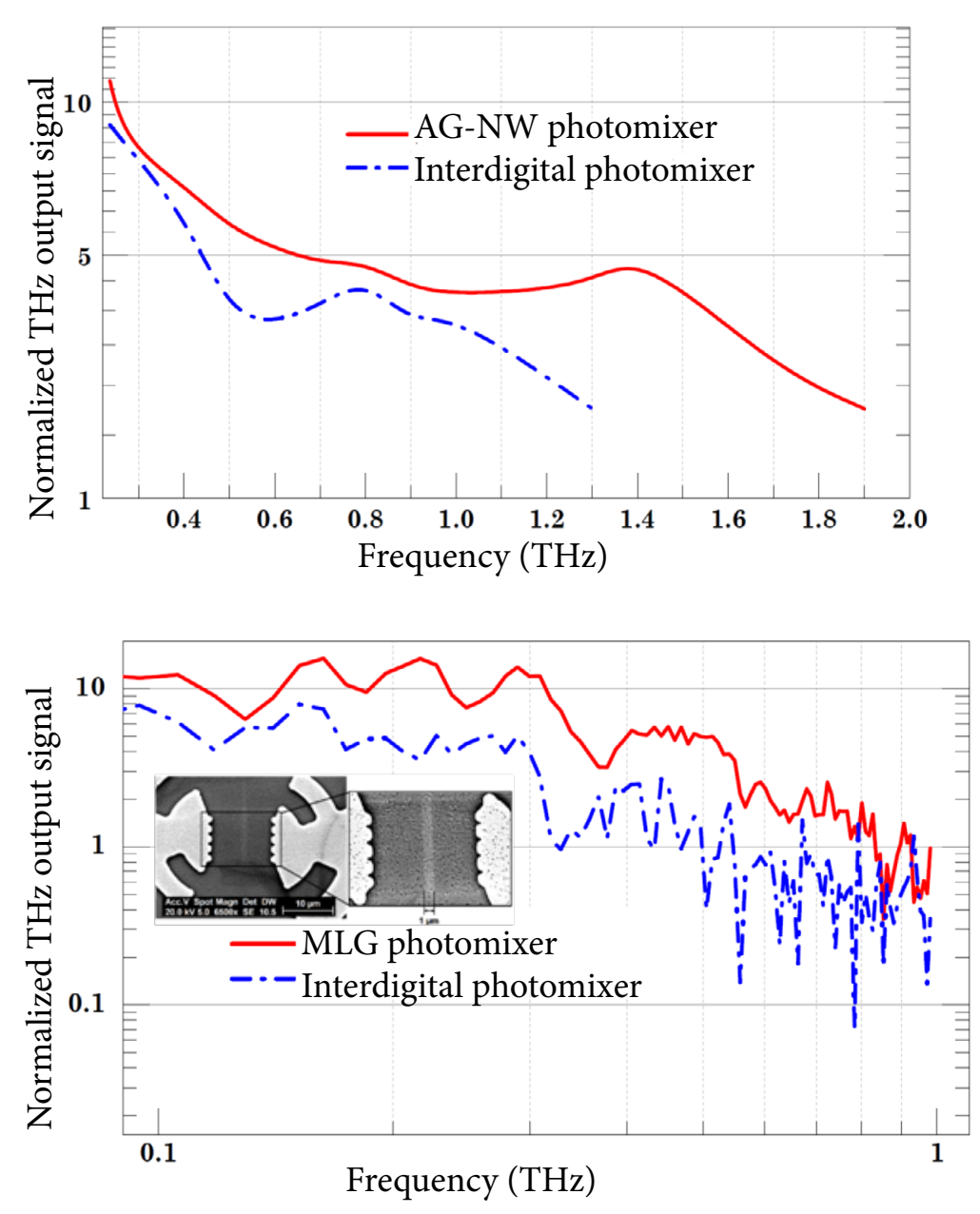

Fig. 4. Comparison of the $\mathrm{THz}$ measurement results between the Ag-NW (given in Fig. 1(a)) and the interdigital photomixer.
Fig. 5. Comparison of the $\mathrm{THz}$ measurement results between the MLG (shown in the inset) and the interdigital photomixer. 
design. A new antenna design is required for this 1-D photomixer configuration to extend the cutoff frequency to $2 \mathrm{THz}$. The 2-D photomixer with MLG (six to eight layers) had a single-gap electrode with $10 \mu \mathrm{m}$ width (simple configuration) providing a larger $\mathrm{THz}$ output power than interdigital six-finger electrodes. The field of plasmonics requires now many new concepts in engineering applications in order to exploit many opportunities of optics and $\mathrm{THz}$ frequencies. The new nanoelectrode photomixers with Ag-NW and graphene can be optimized by multi-finger nanostructures for enhanced photocarrier concentration by the plasmonic effects.

\section{References}

[1] M. Tonouchi, Cutting-edge terahertz technology, Nature Photon. 1(2), 97-105 (2007).

[2] V. Giannini, A. Berrier, S.A. Maier, J.A. SánchezGil, and J.G. Rivas, Scattering efficiency and near field enhancement of active semiconductor plasmonic antennas at terahertz frequencies, Opt. Express 18(3), 2797-2807 (2010).

[3] S.R.K. Rodriguez, Y. Chen, T.P. Steinbusch, M.A. Verschuuren, A.F. Koenderink, and J. Gómez Rivas, From weak to strong coupling of localized surface plasmons to guided modes in a luminescent slab, Phys. Rev. B 90, 235406 (2014).

[4] T.P. Steinbusch, H.K. Tyagi, M.C. Schaafsma, G. Georgiou, and J. Gómez Rivas, Active terahertz beam steering by photo-generated graded index gratings in thin semiconductor films, Opt. Express 22, 26559-26571 (2014).

[5] A. Abass, S.R.K. Rodriguez, T. Ako, T. Aubert, M.A. Verschuuren, D. van Thourhout, J. Beeckman,
Z. Hens, J. Gómez Rivas, and B. Maes, Active liquid crystal tuning of plasmonic enhanced light emission from colloidal quantum dots, Nano Lett. 14, 5555-5560 (2014).

[6] M. Tamagnone, J.S. Gómez-Díaz, J.R. Mosig, and J. Perruisseau-Carrier, Reconfigurable terahertz plasmonic antenna concept using a graphene stack, Appl. Phys. Lett. 101, 214102 (2012).

[7] H.L. Hartnagel, Semiconductor Plasma Instabilities (Heinemann Educational Books LTD, London, 1969).

[8] G.V. Naik and A. Boltasseva, Semiconductors for plasmonics and metamaterials, Phys. Status Solidi RRL 4(10), 295-297 (2010).

[9] M. Obradov, Z. Jakšić, and D. Vasiljević-Radović, Suppression of noise in semiconductor infrared detectors using plasmonics, J. Opt. 16, 125011 (2014).

[10]T. Taliercio, V. Ntsame Guilengui, L. Cerutti, J.B. Rodriguez, and E. Tournié, All-semiconductor plasmonics for mid-IR applications, Proc. SPIE 8807, 880702 (2013).

[11]S. Al-Daffaie, O. Yilmazoglu, F. Küppers, and H. Hartnagel, 1-D and 2-D nanocontacts for reliable and efficient terahertz photomixers, IEEE Trans. Terahertz Sci. Technol. 5(3), 398-405 (2015).

[12] H.L. Hartnagel and S. Preu, in: Proceedings of WOCDICE/EXMATEC 2014 Workshop (Delphi, Greece, 2014).

[13]H.L. Hartnagel, D.S. Ong, and S. Al-Daffaie, in: Proceedings of the 37th Workshop on Compound Semiconductor Devices and Integrated Circuits (WOCSDICE) 2013 (Warnemünde, Germany, 2013).

\title{
PLAZMONIKA PUSLAIDININKIUOSE IR SUBMIKRONINĖSE SIDABRO VIELOSE BEI GRAFENE
}

\author{
H. Hartnagel, S. Al-Daffaie, O. Yilmazoglu
}

Darmštato technikos universitetas, Darmštatas, Vokietija 